Document downloaded from:

http://hdl.handle.net/10251/40477

This paper must be cited as:

Serrano Cruz, JR.; Olmeda González, PC.; Tiseira Izaguirre, AO.; García-Cuevas González, LM.; Lefebvre, A. (2013). Theoretical and experimental study of mechanical losses in automotive turbochargers. Energy. 55:888-898. doi:101016/j.energy.2013.04.042.

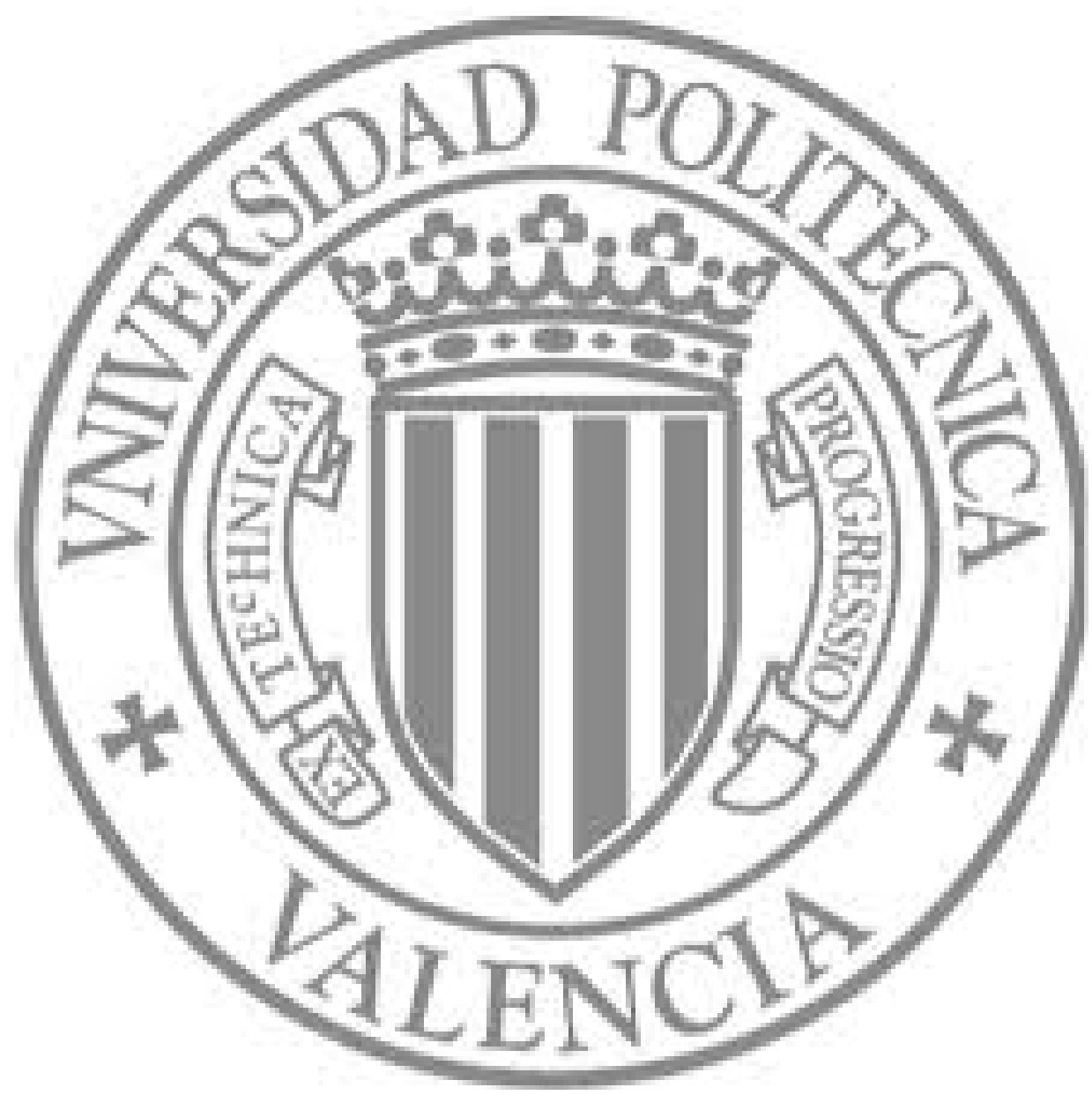

The final publication is available at

http://dx.doi.org/10.1016/j.energy.2013.04.042

Copyright Elsevier 


\title{
Theoretical and experimental study of mechanical losses in automotive turbochargers
}

\author{
J.R. Serrano, P. Olmeda*, A. Tiseira, L.M. García-Cuevas \\ CMT-Motores Térmicos, Universitat Politècnica de València, Camino de Vera s/n. 46022 València, Spain
}

Alain Lefebvre

Renault S.A.

\begin{abstract}
The aim of the present work is to show an approximation, through an experimental an theoretical study, to quantify the mechanical losses in a turbocharging system. These are linked to the dynamics in the turbo shaft bearings, both axial and radial. Theoretical and experimental methodologies are presented in order to develop a mechanical losses model. The experimental work consists on a measurement campaign in quasi-adiabatic operating conditions, while in the theoretical part, a mathematical model is developed taking into account the radial and axial bearings. The model uses some assumptions in order to solve the Navier-Stokes equations, leading to a simplified model which includes viscosity and the Reynolds number of the oil film formed on the bearings. The proposed model has shown a good agreement with the experimental data.
\end{abstract}

Keywords: turbocharger, mechanical losses

\section{Introduction}

Nowadays internal combustion engines, ICE, are facing with two main problems, the pollutants emission and the fuel consumption reduction, in other to fulfill new regional regulations such as the European norm Euro VI [1] while maintaining the engine performance. The new engine design methodology applied to reach these objectives refers to reduce the engine size incrementing the inlet pressure, an action known as downsizing. Usually, this is done using a turbocharger placed in the intake and in the exhaust line. Engine efficiency largely depends on the turbocharger efficiency.

This last parameter depends on the capacity to transform the energy of the engine exhaust gases to mechanical energy by the turbine and then transform this energy to increment the admission pressure by the compressor work. Unfortunately, some of the mechanical energy is lost in the transmission shaft and the systems attached to it as lubrication, bearings and others mechanisms placed in the turbocharger core. The energy loss between the turbine and compressor is called mechanical losses. Recent works, devoted to study this energy loss, discuss about the main difficulties to determine a characteristic value. Two important reasons are found. The turbochargers have a small size and a high rotating speed [2].

Traditionally, the study about turbochargers is focused in medium to high engine loads at low or medium engine speeds due to evaluate the engine on the urban cycle. In this operation condition the average value of compression ratio is around 1.5. In this situation, when the lubricating oil is hot, the mechanical losses are small compared to other energies flowing through the turbocharger.

\footnotetext{
*Corresponding author. Tel: $+34963877650 ;$ fax: +34963877659

Email address: pabolgon@mot.upv.es (P. Olmeda)

URL: www. cmt.upv.es (P. Olmeda)
} 
The importance to homologate the engine in the urban cycle has led to improve the turbocharger performance. In low load cases heat and friction losses can be very important because their magnitude can reach a value similar to the compressor mechanical power [3]. Therefore, it is important to quantify the value of the mechanical losses.

Theoretical studies, using fluid dynamics models (CFD), are done considering the importance of the oil temperature to evaluate the energy losses process. Such studies consider the change of the oil temperature passing through the bearings or the isothermal process [4], finding that a better solution is obtained with the first approximation. Lihua et al. [5] study the design of the hydrostatic spindle, arriving to the conclusion that not only the temperature affects the losses: the other important parameter will be the oil pressure distribution across the journal. Chen [6] studies the rotordynamic characteristics of large turbochargers, but no indications for small turbochargers are mentioned.

Experimental studies about turbocharger mechanical losses are performed in cold tests where the temperatures corresponding to the gas (turbine), oil, and air (compressor) are kept almost constant and equal [7]. With this methodology the heat transfer effect is usually neglected. Therefore, the mechanical power can be determined by means of oil temperature dop. A torquemeter is implemented in [8], but in this case a very complex modification is done to connect the sensor with the turbocharger shaft. Due to the system change it is necessary to isolate the contribution of the torquemeter connection.

Payri et al. [9] explain an empirical model, showing the dependency between the mechanical losses and some dimensionless numbers: Reynolds, Prandtl, and a dimensionless pressure difference, which expression was based on the work of $\mathrm{Hu}$ et al. [10]. The last contribution define the axial thrust in the turbocharger as the pressure difference between the end of the compressor impeller and the turbine backplate, which is related to the outlet of the compressor rotor and the inlet of the turbine rotor. Povedin [8] by means of cold tests and a torquemeter define the importance of the variations of oil pressure and its relation with the variation of the oil mass flow and axial thrust. Deligant et al. [11] used a torquemeter and a magnetic axial load in order to study the load influence on friction power. The results of these tests accounted for up to $12 \%$ of the friction power, so the authors considered less important to implement in a model its influence. Besides, in this work the dependency with oil pressure is estimated small respect to the effect of oil temperature and its relation with oil viscosity.

From authors knowledge there is not a simple mathematical and physical model that attempt to determine mechanical losses in a turbocharger and could be used in zero or one dimensional models, in which the calculation time is an imperative. Therefore, this work presents a physical model obtained by solving the Navier-Stokes equations applying assumptions, valid for turbochargers of ICE, to simplify them. This model has been adjusted with experimental data obtained from a specific turbocharger test bench, in which a cold test campaign similar to that described by Shaaban et al. [7] and Payri et al. [9] was performed.

The first part of this paper concerns about the experimental methodology and the main parameters measured to characterize mechanical losses. Then, the physical model of both the radial and axial bearings are presented and using some assumptions a simplified expression is obtained. After that, results are presented and, finally the main conclusions of the work are outlined.

\section{Analysis of experimental results}

\subsection{Experimental methodology}

Figure 1 shows the layout of a continuous air flow test bench [9]. It is composed by the following parts:

- A screw compressor with a maximum mass flow capacity of $9.84 \mathrm{~m}^{3} / \mathrm{min}$, at a maximum discharging pressure of 3.5 bar (gauge), which provides the mass flow to the turbine. The mass flow rate is controlled by the screw compressor speed or an electronic discharge valve (placed after the screw compressor). The valve is used when a mass flow lower than the minimum supplied by the screw compressor is required. The extra flow is directly discharged to the atmosphere.

- The mass flow is heated in parallel by five tube-type electrical heaters. The flow through each of the heaters is regulated and balanced by means of valves placed on the heater's inlet ports. This system can reach up to 720 $\mathrm{K}$ at the maximum mass flow rate. This hot flow is collected in a plenum and conducted to the turbine inlet. 
- After passing through the turbine, the air is cooled by means of a heat exchanger to allow the mass flow measurement by high accuracy hot film flow meters. All flow meters in the installation have been previously calibrated.

- The compressor sucks air from the atmosphere. The air passes first through a filter and then its flow rate is measured. Downstream of the compressor, there is an electronically driven backpressure valve.

- An independent lubrication system is used to control oil flow rate, pressure (by means of an oil pump and a pressure control valve) and temperature (by using an electrical heater and a cooler). The oil mass flow rate is measured by means of a coriolis flow meter. Lubrication inlet and outlet temperatures are measured by means of low uncertainty platinum resistance temperature detectors. The independent lubrication system is acconditioned to take periodic samples of oil in order to characterize its properties (viscosity, density and specific heat capacity variations with temperature).

- Temperature and pressure sensors are installed on the inlet and outlet pipes of the compressor and the turbine according to SAE J1826 standards [12, 13].

In order to decouple mechanical losses and heat transfer in the turbocharger, the tests has been designed in order to mitigated the influence of heat transfer. These have been performed in the following ways:

- First, temperature drops across the turbocharger has been kept as low as possible. This was achieved by means of the called quasi-adiabatic conditions, that is imposing the same temperature at turbine inlet, lubrication oil inlet and compressor outlet (i.e. $T_{3}=T_{\text {oil,in }}=T_{2}$ ). The main problem of this procedure is the maximum temperature of the lubricating oil, so only low to medium turbocharger compression ratios can be tested as figure 2 shows.

- Secondly, the whole turbocharger has been insulated, so heat losses to the ambient have been minimized.

With these two actions, the turbocharger behavior is expected to be near adiabatic [14, 15]. In this way, the energy absorbed by the lubrication oil will mainly come from the heat generated by friction, i.e. mechanical losses. Albeit conducting the experimental campaign in quasi-adiabatic conditions, the spurious internal heat transfer is computed and the mechanical losses power is corrected following the methodology described by Serrano et al. [16, 17] .

The main limit associated to these kind of tests is the range of compressor points where the test requirements can be accomplished. Figure 2 shows the range of the quasi-adiabatic tests on a full compressor map. The limit is imposed by compressor outlet temperatures higher than $420 \mathrm{~K}$, a condition that can deteriorate the lubricating oil.

This work presents an study about three different turbochargers, whose main characteristics are presented in table 1. Tests in the three turbochargers were performed trying to keep the conditions of quasi-adiabatic tests (i.e. $T_{3}=$ $T_{\text {oil,in }}=T_{2}$ ), tables 2, 3 and 4 show the mean differences between the turbine inlet and compressor outlet temperatures and the oil inlet temperature. As tables 2,3 and 4 show, the measurements performed go from $30 \mathrm{krpm}$ to $190 \mathrm{krpm}$ in steps of $20 \mathrm{krpm}$ in compressor corrected speed for the three tested turbochargers between surge and choke (between 7 and 13 points per iso corrected speed line). At minimum compression ratios (i.e. $30 \mathrm{krpm}$ and $50 \mathrm{krpm}$ ), the quasi-adiabaticity is difficult to achieve [18].

\subsection{Experimental study}

All performed measurements are processed in order to obtain mechanical power and mechanical efficiency. Since tests were quasi-adiabatic, heat losses can be usually neglected [9]. Nevertheless, they are taken into account as found in [16, 17] :

$$
\dot{W}_{m l} \simeq \dot{m}_{o i l} \cdot c \cdot \Delta T_{o i l}-\dot{Q}_{o i l}
$$

while mechanical efficiency can be obtained as:

$$
\eta_{\text {mech }}=\frac{\dot{W}_{\text {comp }}}{\dot{W}_{\text {turb }}}=\frac{\dot{W}_{\text {turb }}-\dot{W}_{m l}}{\dot{W}_{\text {turb }}}=1-\frac{\dot{W}_{m l}}{\dot{W}_{\text {turb }}}
$$




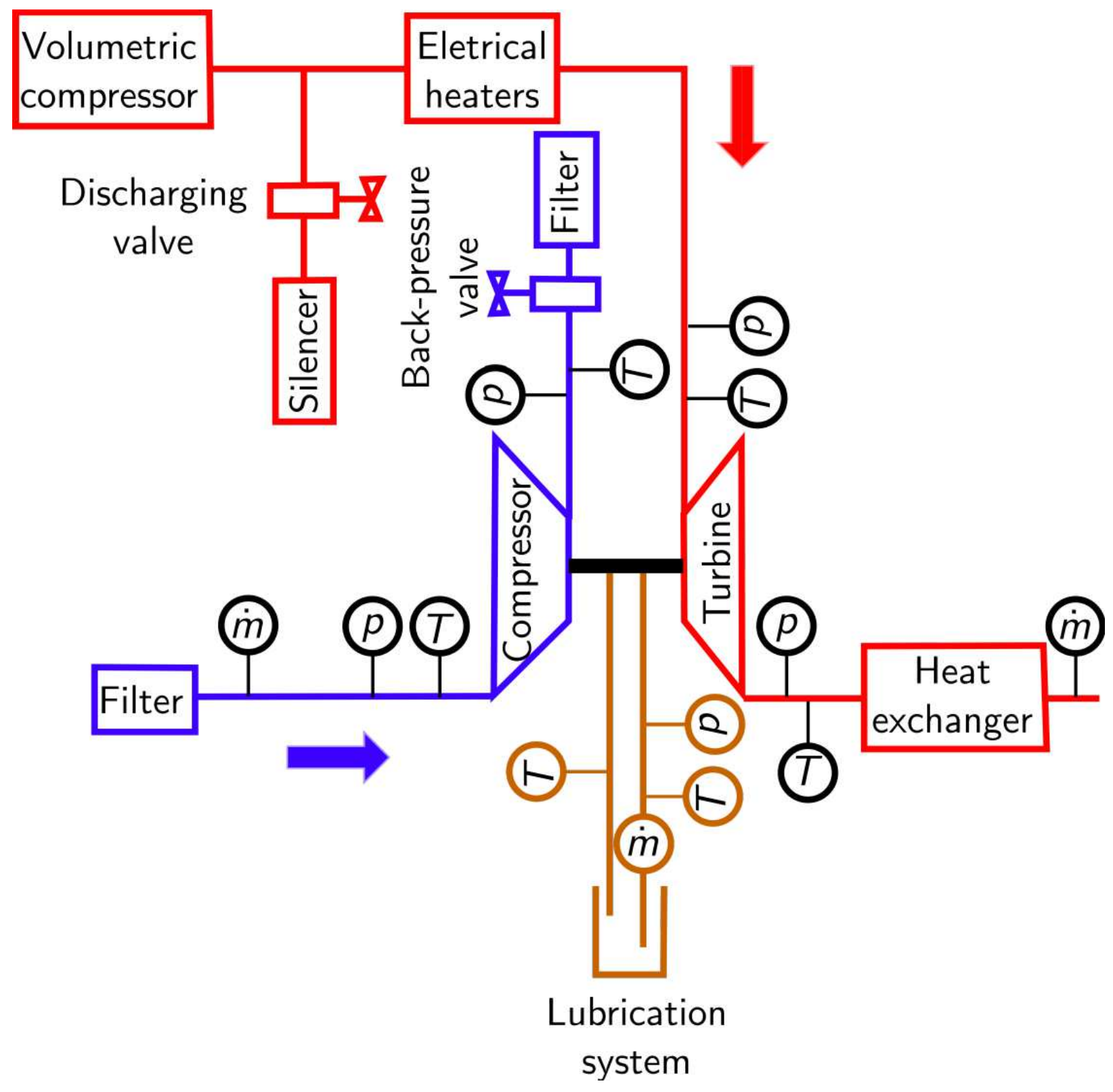

Figure 1: Schematic test bench and location of main sensors 


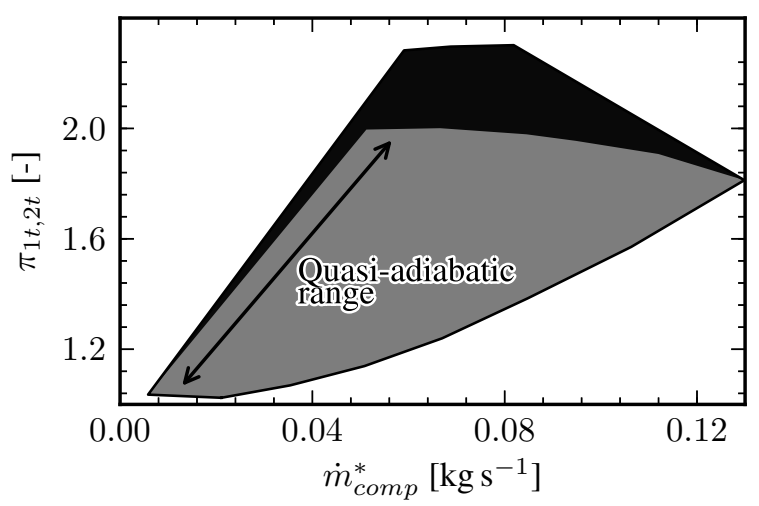

Figure 2: Quasi-adiabatic measurement range in compressor map

\begin{tabular}{|c|c|}
\hline \multicolumn{2}{|l|}{ First turbocharger } \\
\hline Turbine wheel diameter [mm] & 41 \\
\hline Compressor wheel diameter [mm] & 49 \\
\hline VGT & yes, vanes \\
\hline Water cooled & yes \\
\hline Type of journal bearing & fixed \\
\hline Engine power $[\mathrm{kW}]$ & 129 \\
\hline Engine type & diesel \\
\hline Displacement [1] & 2.0 \\
\hline \multicolumn{2}{|l|}{ Second turbocharger } \\
\hline Turbine wheel diameter [mm] & 38 \\
\hline Compressor wheel diameter $[\mathrm{mm}]$ & 46 \\
\hline VGT & yes, vanes \\
\hline Water cooled & no \\
\hline Type of journal bearing & floating ring \\
\hline Engine power $[\mathrm{kW}]$ & 96 \\
\hline Engine type & diesel \\
\hline Displacement [1] & 1.6 \\
\hline \multicolumn{2}{|l|}{ Third turbocharger } \\
\hline Turbine wheel diameter [mm] & 36.5 \\
\hline Compressor wheel diameter [mm] & 40 \\
\hline VGT & no \\
\hline Water cooled & yes \\
\hline Type of journal bearing & floating ring \\
\hline Engine power $[\mathrm{kW}]$ & 75 \\
\hline Engine type & petrol \\
\hline Displacement [1] & 1.2 \\
\hline
\end{tabular}

Table 1: Main characteristics of the employed turbochargers 


\begin{tabular}{cccc}
\hline $\begin{array}{c}n \\
{[\mathrm{rpm}]}\end{array}$ & $\begin{array}{c}\overline{T_{\text {oil,in }}} \\
{[\mathrm{K}]}\end{array}$ & $\begin{array}{c}\overline{T_{2}-T_{\text {oil,in }}} \\
{[\mathrm{K}]}\end{array}$ & $\begin{array}{c}\overline{T_{3}-T_{\text {oil,in }}} \\
{[\mathrm{K}]}\end{array}$ \\
\hline 30000 & 299.8 & -1.6 & 2.4 \\
50000 & 305.7 & 0.3 & 3.3 \\
70000 & 317.1 & 1.1 & 2.5 \\
90000 & 333.1 & 1.7 & 3.1 \\
110000 & 349.2 & 0.6 & 1.3 \\
130000 & 369.7 & 1.5 & 1.1 \\
150000 & 397.4 & 3.7 & 0.3 \\
170000 & 417.2 & 12.6 & 1.5 \\
\hline
\end{tabular}

Table 2: Mean deviations the adiabatic temperatures. First turbocharger.

\begin{tabular}{cccc}
\hline$n$ & $\overline{T_{\text {oil,in }}}$ & $\overline{T_{2}-T_{\text {oil,in }}}$ & $\overline{T_{3}-T_{\text {oil,in }}}$ \\
{$[\mathrm{rpm}]$} & {$[\mathrm{K}]$} & {$[\mathrm{K}]$} & {$[\mathrm{K}]$} \\
\hline 30000 & 297.0 & -3.1 & 4.9 \\
50000 & 298.8 & -0.2 & 0.8 \\
70000 & 310.3 & -1.7 & 4.3 \\
90000 & 321.2 & 0.6 & 1.0 \\
110000 & 343.7 & -9.0 & 0.7 \\
130000 & 357.8 & -1.7 & 1.5 \\
150000 & 379.0 & 3.1 & 2.0 \\
170000 & 400.2 & 3.6 & 1.4 \\
\hline
\end{tabular}

Table 3: Mean deviations the adiabatic temperatures. Second turbocharger.

\begin{tabular}{|c|c|c|c|}
\hline $\begin{array}{c}n \\
{[\mathrm{rpm}]}\end{array}$ & $\begin{array}{c}\overline{T_{\text {oil,in }}} \\
{[\mathrm{K}]}\end{array}$ & $\begin{array}{c}\overline{T_{2}-T_{\text {oil,in }}} \\
{[\mathrm{K}]}\end{array}$ & $\begin{array}{c}\overline{T_{3}-T_{\text {oil,in }}} \\
{[\mathrm{K}]}\end{array}$ \\
\hline 50000 & 305.6 & 1.4 & 4.1 \\
\hline 70000 & 314.1 & 1.0 & 4.3 \\
\hline 90000 & 322.2 & 0.1 & 3.0 \\
\hline 110000 & 334.4 & -1.6 & 2.6 \\
\hline 130000 & 345.3 & 2.0 & 0.6 \\
\hline 150000 & 362.3 & 5.0 & 1.8 \\
\hline 170000 & 386.0 & -0.3 & -0.6 \\
\hline 190000 & 403.4 & 3.0 & 3.3 \\
\hline
\end{tabular}

Table 4: Mean deviations the adiabatic temperatures. Third turbocharger. 
Figure 3 shows the mechanical efficiency obtained for all three turbochargers in a compressor map, where a clear relationship between this efficiency and the compressor load is observed, i.e. the higher the load, the higher mechanical efficiency. For low to medium compression ratios (as the tests were performed with the constraints explained in section 2.1. the maximum measured load was approximately one third of the turbochargers maximum load), the value of this efficiency is higher than a $90 \%$. So, for higher loads and following the trends show in figure 3 , maximum efficiencies are expected. On the other hand, minimum measured efficiencies down to a 50\% at very low compression ratios, that is when the engine is at iddle or at very low load that corresponds to a high number of operating conditions in the urban cycle [1].

So, in these low load points the use of a fixed mechanical efficiency will lead to a very high error in modeling the turbocharger behavior, and hence if a proper model of the turbocharger is expected, it is imperative to model as proper as possible this efficiency (or mechanical losses).

Finally, it must be mentioned that oil viscosity has played an important role in the obtained values of mechanical efficiency, that is at low compression ratios (due to the constraints in the measurements) oil temperatures are lower than those used at medium compression ratios. On the contrary, these oil temperatures are higher than those used in the beginning of the tests of the European cycle [19].
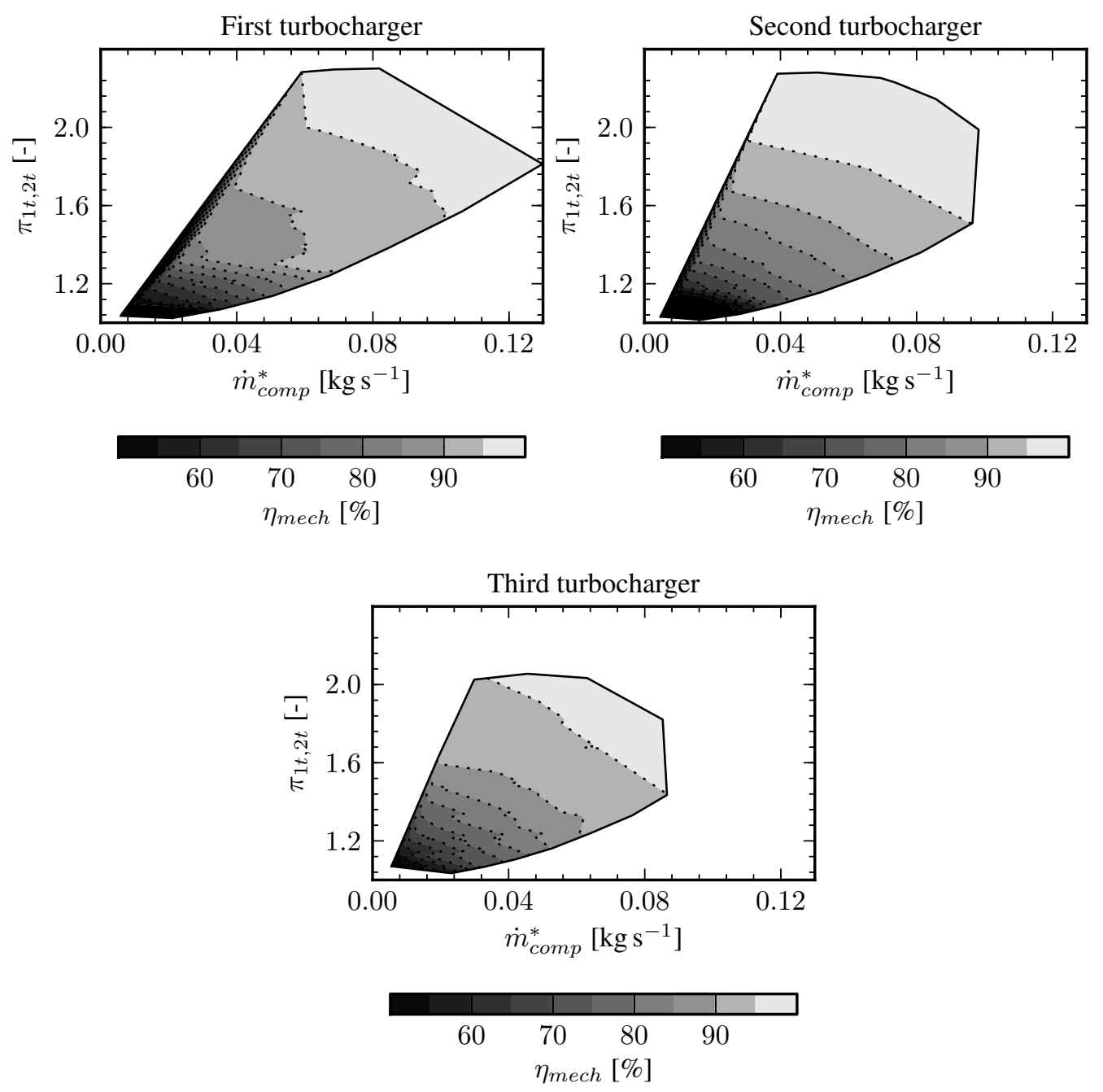

Figure 3: Measured mechanical efficiencies 


\section{Model description}

In small automotive turbochargers, the bearing system usually consists in two different plain bearings: a radial journal bearing and a thrust bearing. Therefore, the mechanical losses can be splitted into two different parts:

$$
\dot{W}_{m l}=\dot{W}_{j b}+\dot{W}_{t b}
$$

The first term, $\dot{W}_{j b}$, is due to the power losses in the radial bearing, where the bush can be fixed or free. The second term, $\dot{W}_{t b}$, is due to the power losses in the thrust bearing. In both bearings, there is a tangential speed gradient in the oil film that creates viscous stresses, thus heating the oil and creating a loss in the power transmitted from the turbine to the compressor.
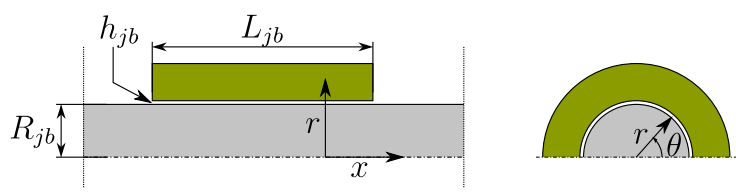

Figure 4: Schematic model of a journal bearing

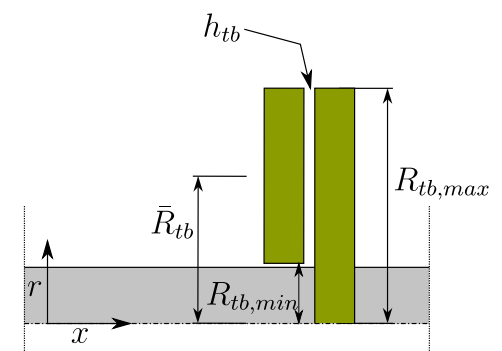

Figure 5: Schematic model of a thrust bearing

To obtain the power losses in the bearing system, the behavior of the oil in an ideal journal and thrust bearing is studied. The equations to compute the power losses in the ideal bearings (figures 4 and 5 , are developed performing the following assumptions:

1. Oil behaves as incompressible fluid.

2. The flow through the bearing is steady.

3. The bearing is sufficiently long to considerer constant behavior on each section

4. The flow is circumferentially symmetric.

5. Body forces are negligible.

6. The Reynolds number $R e=\frac{\rho u_{c} h}{\mu}$ is expected to be small enough to make the viscous stresses comparable to the inertial forces of the fluid.

7. Film thickness in the bearing is smaller than any bearing geometry, i.e.

(a) $\frac{h_{j b}}{L_{j b}} \ll 1, \frac{h_{j b}}{R_{j b}} \ll 1$ and therefore $\frac{\rho u_{c} h_{j b}^{2}}{\mu L_{j b}} \ll 1$

(b) $\frac{h_{t b}}{R_{t b}} \ll 1 \Rightarrow \frac{\rho u_{c} h_{t b}^{2}}{\mu R_{t b}} \ll 1$

In the analyzed turbochargers, points $67 \mathrm{a}$ and $7 \mathrm{~b}$ were true for the whole dataset, being the results of points $7 \mathrm{a}$ and $7 \mathrm{~b}$ between 0.01 and 0.1 . 


\subsection{Journal bearing model}

The power drawn by the ideal plain journal bearing is approximated by equation 4

$$
\dot{W}_{j b}=n \cdot \int_{A_{j b}} R_{j b} \cdot \tau^{\prime} \cdot \mathrm{d}(\vec{n} A)
$$

being $n$ the rotational speed of the journal, $A_{j b}$ the surface of the journal bearing, $R_{j b}$ the radius, $\tau^{\prime}$ the viscous stresses tensor and $\vec{n}$ the unitary normal vector to the surface. The integral can be approximated by the product of the surface of the journal bearing, $A_{j b}=2 \pi R_{j b} \cdot L_{j b}$, multiplied by the mean value of $\tau^{\prime} \cdot \vec{n}$, multiplied by the tangential speed $R_{j b} \cdot n$ :

$$
\begin{aligned}
\dot{W}_{j b} & =\cdot A_{j b} \cdot \overline{\left(\tau^{\prime} \cdot \vec{n}\right)} \cdot R_{j b} \cdot n \\
& =\left.2 \pi R_{j b} \cdot L_{j b} \cdot \mu\left(\bar{T}_{o i l}\right) \frac{\partial u_{\theta}}{\partial r}\right|_{R_{j b}} \cdot R_{j b} \cdot n
\end{aligned}
$$

To obtain $\frac{\partial u_{\theta}}{\partial r}$, the continuity equation and the conservation of momentum are used. The process is described in Appendix A.1 from equation A.1 to A.7. The obtained value is shown in equation 6

$$
\left.\frac{\partial u_{\theta}}{\partial r}\right|_{R_{j b}} \simeq n \frac{R_{j b}}{h_{j b}}
$$

If the journal bearing is short as seen in figure 6, the oil tangential speed gradient in the center of the bearing (section B-B) is similar to the ideal, but it is almost zero near the edges (section A-A). In this case, a correction factor $k_{j b}$ can be used.

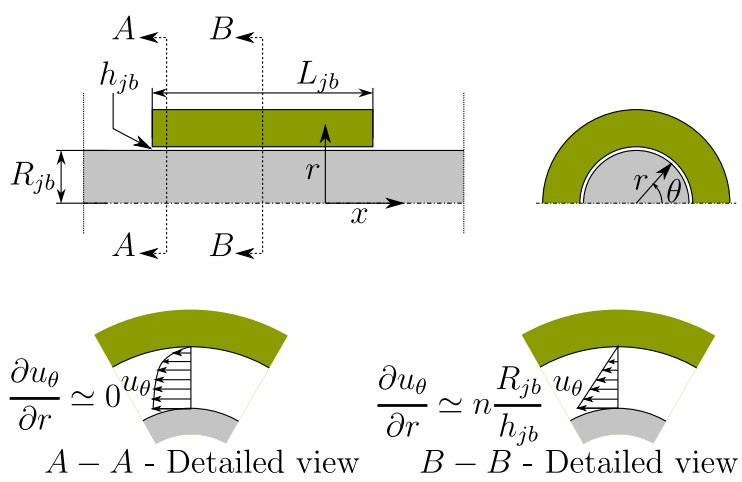

Figure 6: Simplified model of a short journal bearing

Hence, the mechanical power loss in the radial bearing is computed as:

$$
\dot{W}_{j b}=2 \pi R_{j b}^{3} k_{j b} \frac{L_{j b}}{h_{j b}} \mu\left(\bar{T}_{o i l}\right) n^{2}
$$

The geometrical data may be directly measured or fitted experimentally within a simple constant parameter $\left(R_{j b}^{3} k_{j b} L_{j b} / h_{j b}\right)$ if mechanical power data were available. 


\subsection{Thrust bearing model}

In the case of an ideal thrust bearing, the power losses can be computed as:

$$
\dot{W}_{t b}=n \cdot \int_{A_{t b}} r \cdot \tau^{\prime} \cdot \mathrm{d}(\vec{n} A)
$$

Again, the integral of equation 8 can be approximated using mean values. Now, the surface of the thrust bearing is $A_{t b}=\pi\left(R_{t b, \text { max }}^{2}-R_{t b, \text { min }}^{2}\right)$, the mean tangential speed is $\bar{R}_{t b} \cdot n$ and the mean viscous stress is $\mu\left(\bar{T}_{o i l}\right) \frac{\partial u_{\theta}}{\partial x}$ :

$$
\begin{aligned}
\dot{W}_{t b} & =A_{t b} \cdot\left(\overline{\tau^{\prime} \cdot \vec{n}}\right) \cdot \bar{R}_{t b} \cdot n \\
& =\left.\pi\left(R_{t b, \text { max }}^{2}-R_{t b, \text { min }}^{2}\right) \cdot \mu\left(\bar{T}_{o i l}\right) \frac{\partial u_{\theta}}{\partial x}\right|_{\bar{R}_{t b}, h_{t b}} \cdot \bar{R}_{t b} \cdot n
\end{aligned}
$$

where $\left.\frac{\partial u_{\theta}}{\partial x}\right|_{\bar{R}_{t}, h_{t b}}$ can be approximated using the continuity equation and the conservation of momentum equations as described in Appendix A.2 from equation A.8 to A.28. The obtained result of $\left.\frac{\partial u_{\theta}}{\partial x}\right|_{\bar{R}_{t b}, h_{t b}}$ is shown in equation 10

$$
\left.\frac{\partial u_{\theta}}{\partial x}\right|_{\bar{R}_{t b}, h_{t b}}=n \cdot \frac{\bar{R}_{t b}}{\sqrt[3]{\frac{6 k_{m} \dot{m} \varphi \mu}{F_{a t} \rho}}}
$$

where $\varphi$ is computed as shown in equation A.22 and $F_{a t}$, see figure A.1, is shown in equation 11 obtained after solving equations A.23 to A.25

$$
F_{a t}=\left|A_{\text {comp }}^{\prime} \frac{p_{1}-p_{2}}{4}+A_{\text {turb }}^{\prime} \frac{p_{3}^{\prime}-p_{4}}{2}\right|
$$

If the thrust bearing is short, which is usually the case, the tangential speed gradient should be corrected by a constant factor $k_{t b}$ as in the case of the plain radial bearing. The factor $k_{t b}$ can also take into account differences in the speed gradient due to other effects like grooves or gaps in the bearing. Figure 7 shows an scheme of short bearing effects on oil film velocity in the tangential direction.
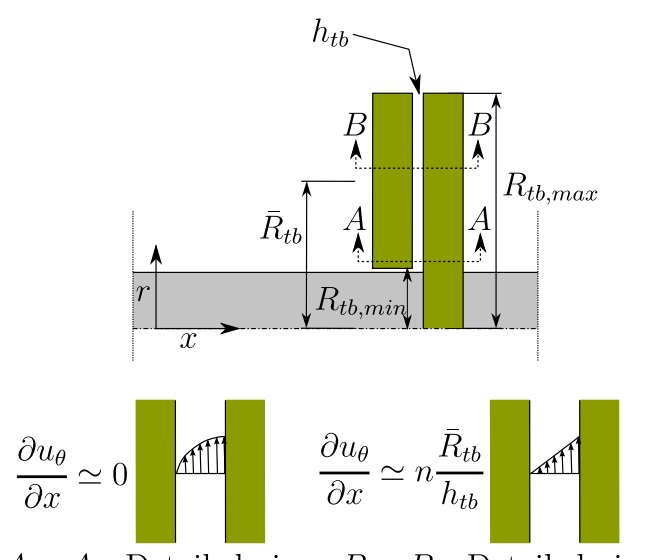

$A-A$ - Detailed view $B-B$ - Detailed view

Figure 7: Simplified model of a short thrust bearing

Therefore, the final expression for the power loss in the thrust bearing is shown in equation 12 .

$$
\dot{W}_{t b}=k_{t b} \pi\left(R_{t b, \text { max }}^{2}-R_{t b, \text { min }}^{2}\right) \cdot \bar{R}_{t b}^{2} \sqrt[3]{\frac{F_{a t} \rho}{6 k_{m} \dot{m} \varphi \mu}} \mu n^{2}
$$




\subsection{Full model}

In both bearings (equations 7 and 12 appears the oil viscosity, which varies considerably when increasing temperature. The power losses computed assuming a constant temperature equal to the inlet temperature are overestimated, as shown in [4]. To compute the relationship between oil viscosity and temperature, Vogel's correlation has been used (see equation 13 , but other correlations may apply.

$$
\mu=\mu(T)=K_{1} \cdot e^{\frac{K_{2}}{T-K_{3}}}
$$

The values of the constants of equation 13 may be obtained by experimental means using viscometers or using manufacturer-provided data sheets.

The value of the mean viscosity of the flow $\left(\mu\left(\bar{T}_{\text {oil }}\right)\right)$ can be obtained supposing that the power losses in the bearings are equal to the leap in enthalpy in the oil in adiabatic contitions.

$$
\begin{aligned}
\dot{W}_{m l} \simeq & \dot{m} c\left(T_{\text {oil,out }}-T_{\text {oil,in }}\right) \\
= & \dot{W}_{j b}+\dot{W}_{t b} \\
= & k_{j b} 2 \pi R_{j b}^{3} \frac{L_{j b}}{h_{j b}} \mu\left(\bar{T}_{o i l}\right) n^{2} \\
& +k_{t b} \pi\left(R_{t b, \max }^{2}-R_{t b, \text { min }}^{2}\right) \\
& \cdot \bar{R}_{t b}^{2} \sqrt[3]{\frac{F_{a t} \rho}{6 k_{m} \dot{m} \varphi \mu\left(\bar{T}_{o i l}\right)}} \cdot \mu\left(\bar{T}_{o i l}\right) n^{2}
\end{aligned}
$$

The weighted mean temperature of the oil is computed as:

$$
\bar{T}_{\text {oil }} \simeq T_{\text {oil, in }}+k_{T}\left(T_{\text {oil,out }}-T_{\text {oil,in }}\right)
$$

Using the relationship between $T_{\text {oil,out }}, \bar{T}_{\text {oil }}$ and $T_{\text {oil,in }}$ in the energy balance equation:

$$
\begin{aligned}
\bar{T}_{o i l} & =T_{o i l, i n}+k_{T} k_{j b} 2 \pi R_{j b}^{3} \frac{L_{j b}}{h_{j b}} \mu\left(\bar{T}_{o i l}\right) \frac{n^{2}}{\dot{m} c} \\
& +k_{T} k_{t b} \pi\left(R_{t b, \text { max }}^{2}-R_{t b, \min }^{2}\right) \\
& \cdot \bar{R}_{t b}^{2} \sqrt[3]{\frac{F_{a t} \rho}{6 k_{m} \dot{m} \varphi \mu\left(\bar{T}_{o i l}\right)}} \cdot \mu\left(\bar{T}_{o i l}\right) \frac{n^{2}}{\dot{m} c}
\end{aligned}
$$

which is an implicit equation that can be solved for $\bar{T}_{\text {oil }}$ using the relation of $\mu(T)$ given in equation 13

Using the value of $\mu\left(\bar{T}_{\text {oil }}\right)$ obtained by solving 16 a non-isothermal approximation of the power losses in the bearing system is obtained.

The values of $K_{1}, K_{2}, K_{3}, c$ and $\rho$ may be obtained using experimental results or consulting the data sheet of the oil used, if it is not very aged. If the density and the specific heat capacity of the oil is expected to change appreciably during the non-isothermal evolution, their behavior should be modeled as with the dynamic viscosity. The authors of this work found good results sopposing $k_{T}$ equal to $0.75 . k_{j b}$ and $k_{t b}$ can be obtained by experimental results. $\varphi$ is computed by equation A.22 and $F_{a t}$ is calculated as shown in equation 11 .

\section{Discussion of the results}

\subsection{Model adjustment}

The developed model in section 3 needs the adjustment of different parameters. The following procedure has been employed: 
- First, a random subset of only one tenth of the whole experimental dataset of each turbocharger were selected.

- Then, the model parameters were adjusted in order to predict mechanical efficiency that fit with the experimental data in three different ways in order to both analyze the behavior of the different parameters under different assumptions and the model sturdiness by means of the variation on the obtained parameters:

- First, considering that there were only a journal bearing, then only one parameter has been fitted: $k_{j b}$ in equation 7

- Second, only a thrust bearing is considered, then three parameters has been fitted: $k_{t b}$ in equation $12, k_{A_{c}}$ in equation A.26 and $k_{A_{t}}$ in equation A.27.

- And the last option is to consider both types of bearings in the model and, therefore four parameters were adjusted: one for the journal bearing and three for the thrust bearing.

The fitting procedure has been made minimizing the root mean square error of the mechanical losses power (equation 17).

$$
\varepsilon_{R M S}=\sqrt{\frac{1}{N} \cdot \sum_{i}^{N}\left(\frac{\dot{W}_{\text {ml,model }_{i}}-\dot{W}_{\text {ml,meas }_{i}}}{\dot{W}_{\text {ml,meas }}}\right)^{2}}
$$

by means of the SLSQP algorithm developed by Kraft [20] using SciPy [21].

\subsection{Model validation}

As it has been mentioned previously, the parameters obtained in the adjustment process with a subset of measurement points (see table 5) have been used to predict mechanical power in the three turbochargers for all the measured points.

Nevertheless, results for the partial models were not satisfactory, so they were fitted to the whole dataset, giving similar bad results, which have been plotted in figure 8. The other results corresponding to the whole model (journal plus thrust bearing) have been plotted in figures 9 and 10 . The parameters $k_{j b}$ and $k_{t b}$ should lie between 0 and 1 and $k_{A_{c}}$ and $k_{A_{t}}$ should be near unity when the model assumptions are valid (see table 5).

The thrust bearing submodel alone (figure 8 left column) does not give satisfactory results at any working conditions. Also, $k_{t b}$ is higher than unity in the three turbochargers (table 5). On the other hand, the radial bearing submodel alone (figure 8 right column) shows good behavior at low compression ratios, when the axial thrust is minimized, but fails at medium and higher compression ratios and high axial force.

The full model gives good results with very little computational cost. Also, the results obtained by fitting a random partial dataset (figure 9) are almost identical to that of figure 10, where the parameters were fitted to the whole experimental database, showing model robustness.

\subsection{Analysis of model parameters}

A linear correlation between the journal bearing geometry and parameter $k_{j b}$ and the area of the compressor wheel was found, as seen in figure 11 . The effect of the momentum of inertia of the shaft and the compressor and turbine wheels is critical to design the radial bearing, what might be the cause of this correlation. Nevertheless, more work would be needed to confirm it.

$k_{A_{c}}$ and $k_{A_{t}}$ are near unity, being it expected if the pressure distribution in the wheels is close to the linear approximation proposed.

$k_{t b}$ is plotted against $R_{t b, \text { max }}-R_{t b, \min }$ in figure 12 . The highest value is for the third turbocharger. Its thrust bearing is shown in figure 14, where no gaps are found in the washer, what could explain the unity value of $k_{t b}$. The second and third turbochargers have lower values, showing a rising tendency with $R_{t b, \max }-R_{t b, \min }$. These low values of $k_{t b}$ can be explained by the presence of gaps in the washer, as seen in figure 13 . However, further research is needed to confirm these trends and to develop general rules to select values of the parameters for simulations in cases where no physical measurement of the mechanical losses are available to fit the model. 
First turbocharger, no journal bearing
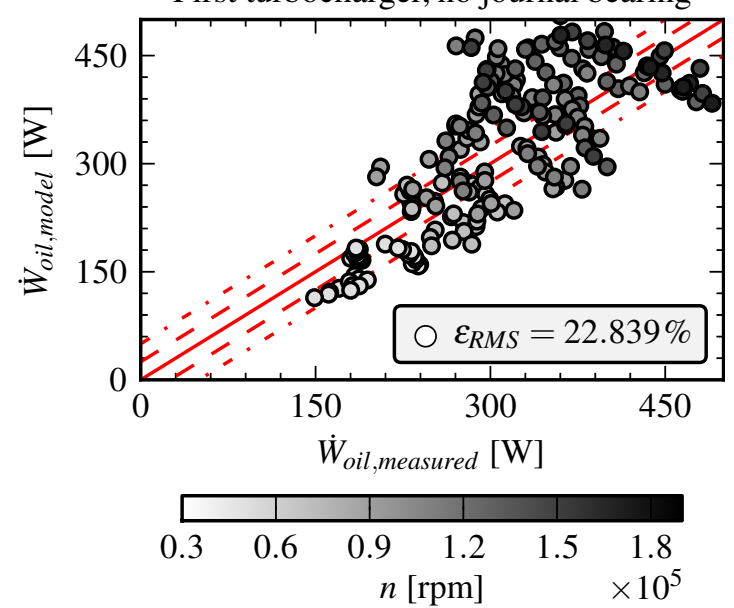

Second turbocharger, no journal bearing
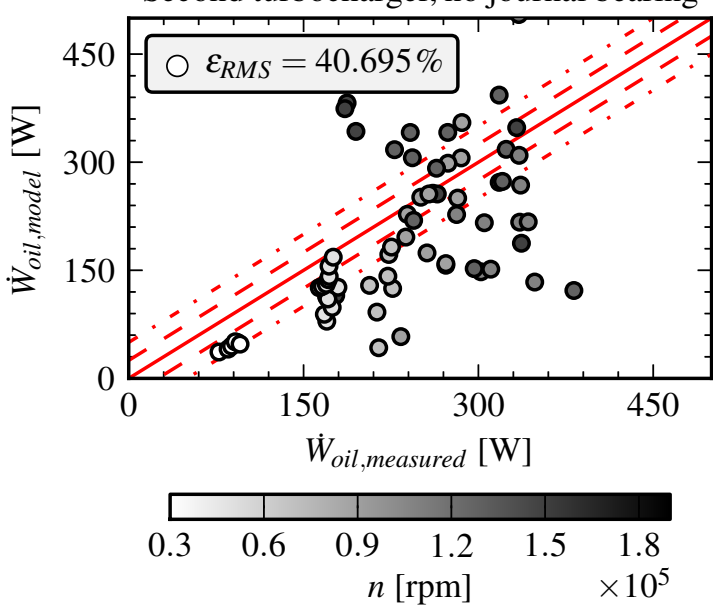

Third turbocharger, no journal bearing

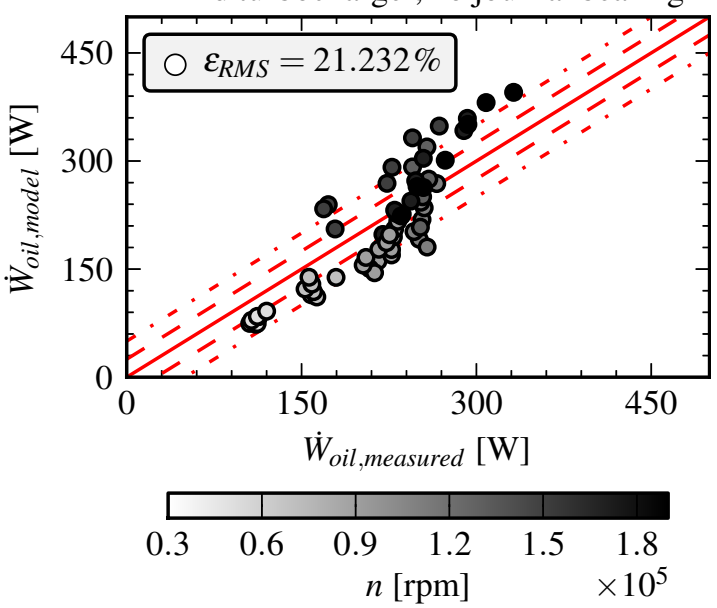

First turbocharger, no thrust bearing
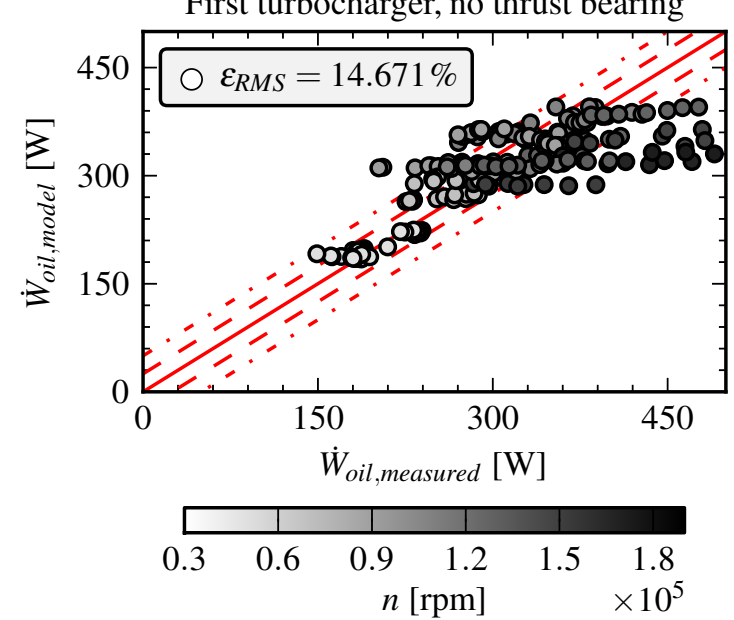

Second turbocharger, no thrust bearing
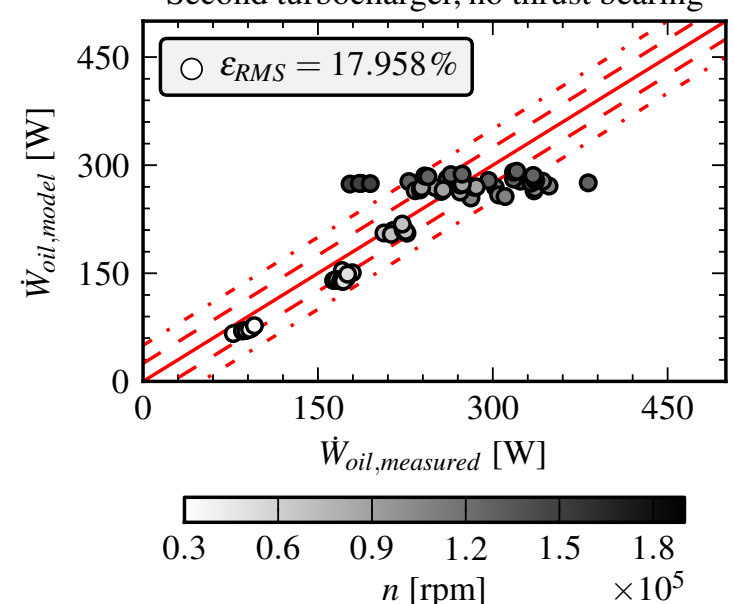

Third turbocharger, no thrust bearing

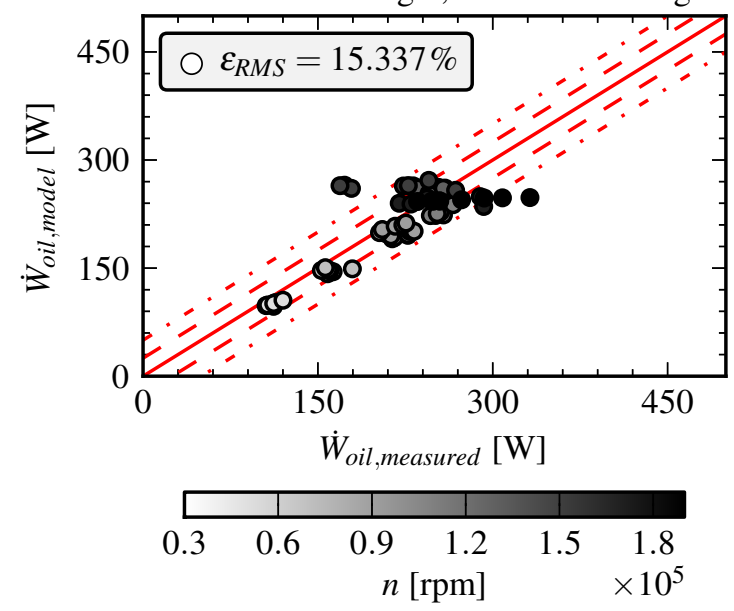

Figure 8: Model results applied to full dataset. The left column is adjusted with no radial bearing effects. The right column is adjusted with no axial bearing effects. 
First turbocharger
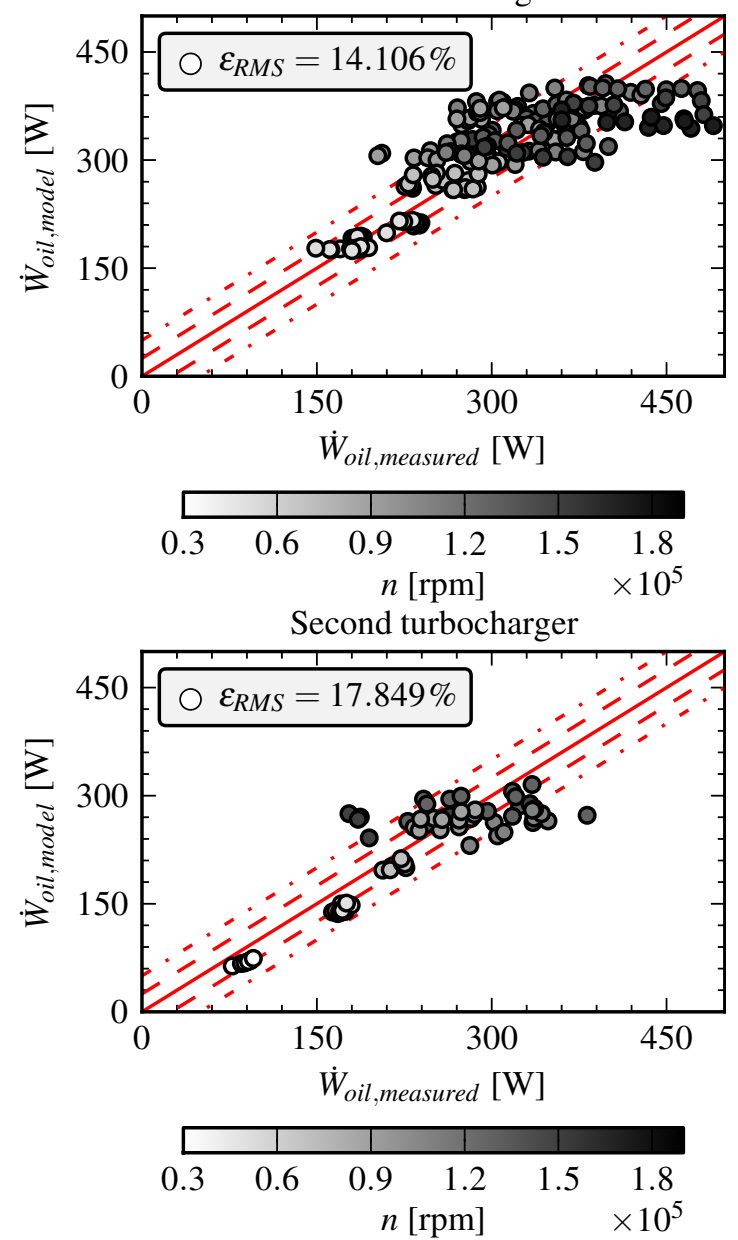

Third turbocharger

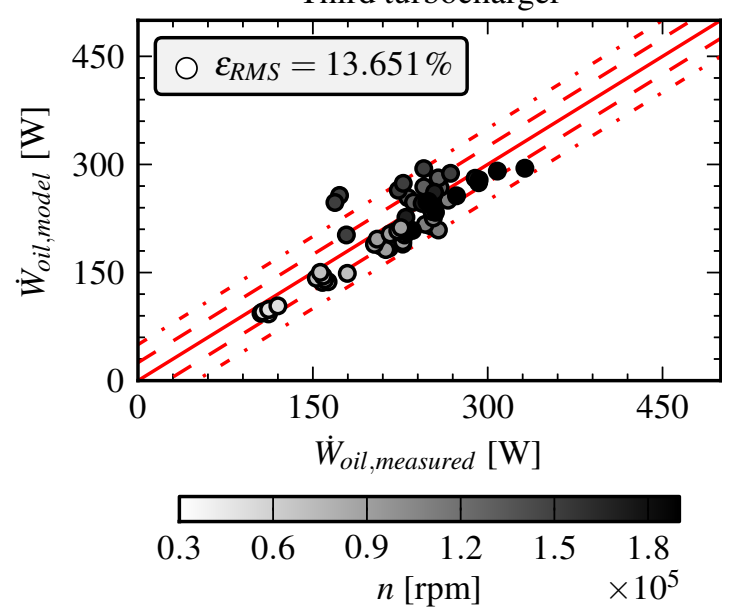

First turbocharger
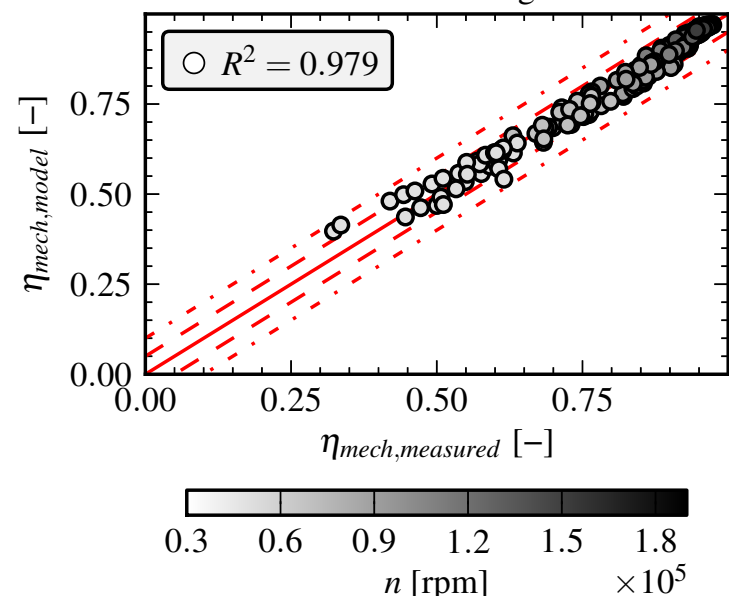

Second turbocharger

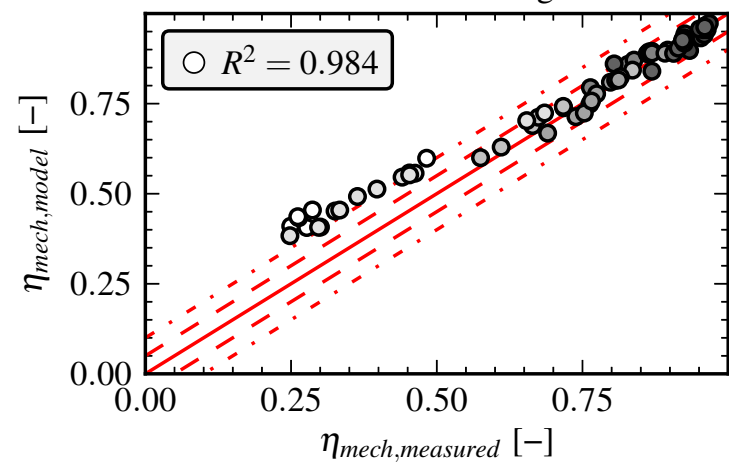

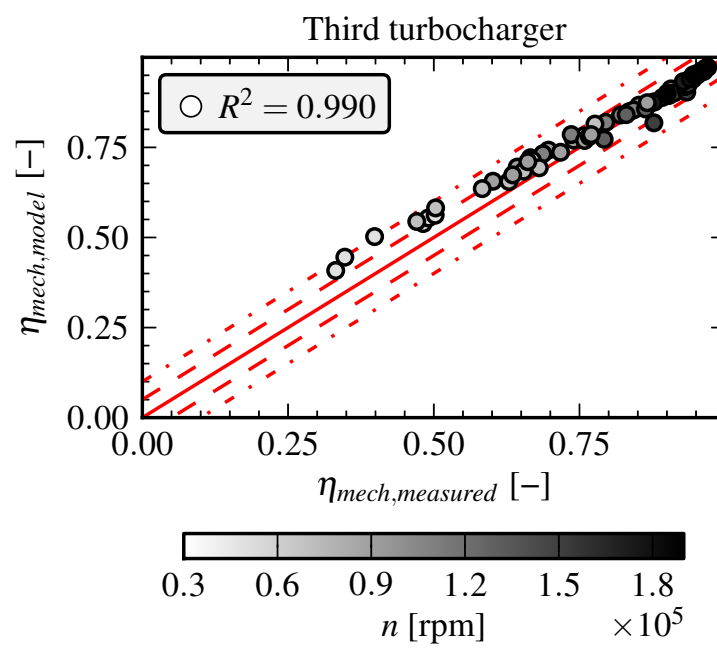

Figure 9: Model results applied to full dataset. The parameters are obtained fitting only a random subset of the experimental data. 
First turbocharger
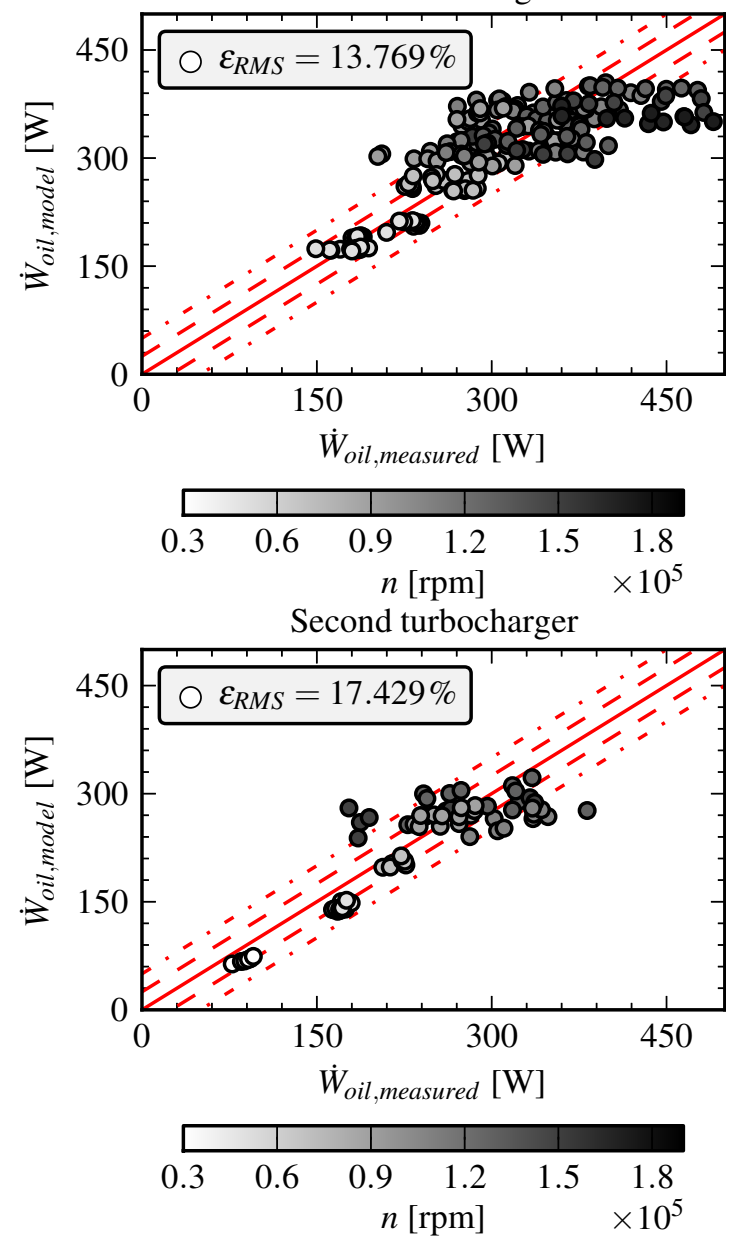

Third turbocharger

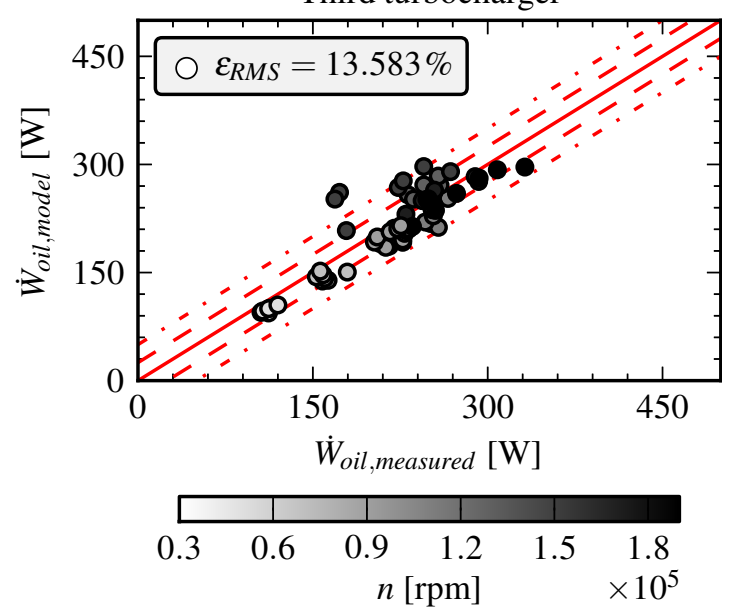

First turbocharger
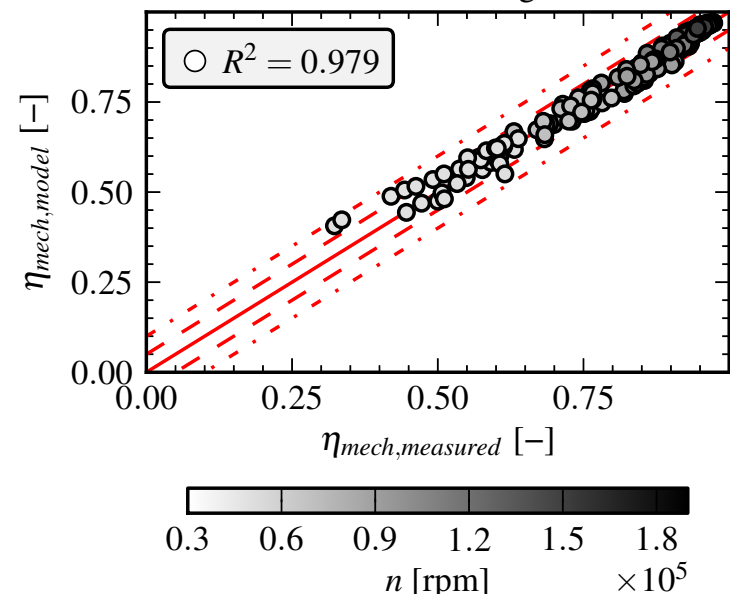

Second turbocharger

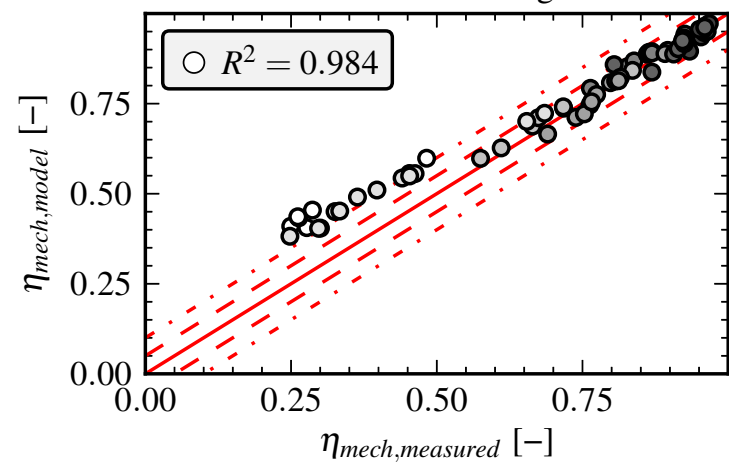

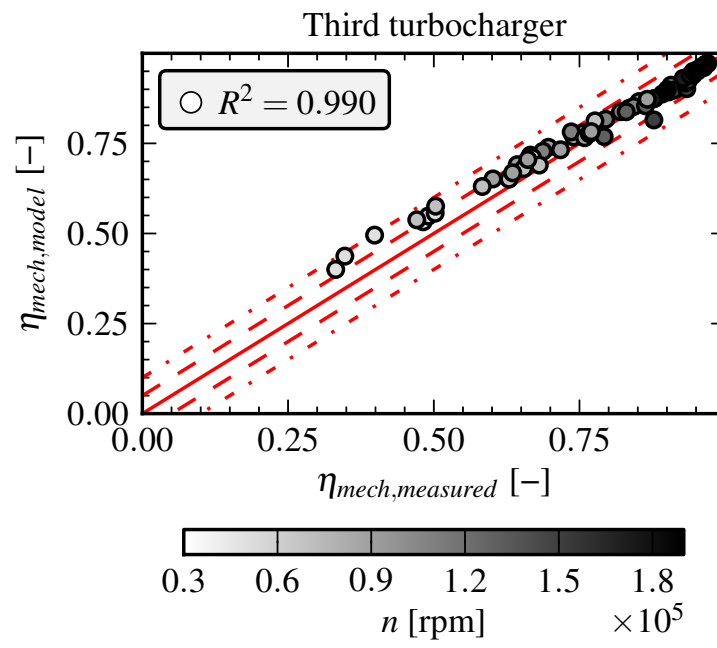

Figure 10: Model results applied to full dataset. The parameters are obtained fitting the whole dataset. 


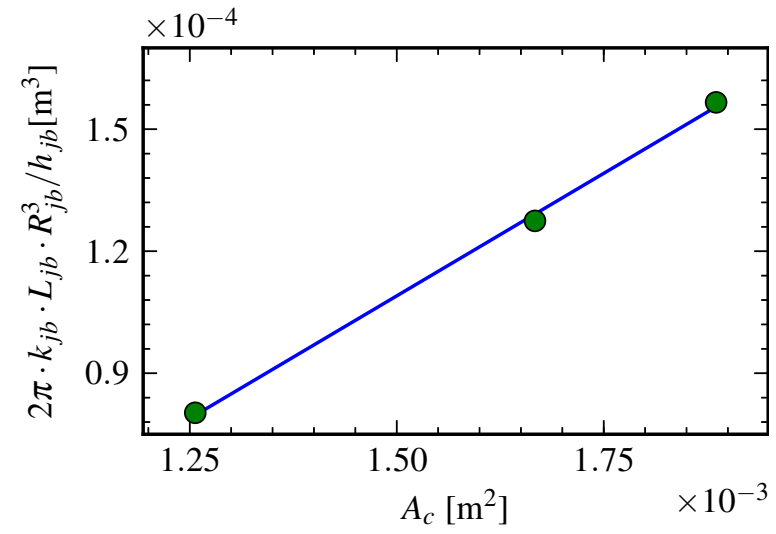

Figure 11: Correlation between the journal bearing model and $A_{c}$

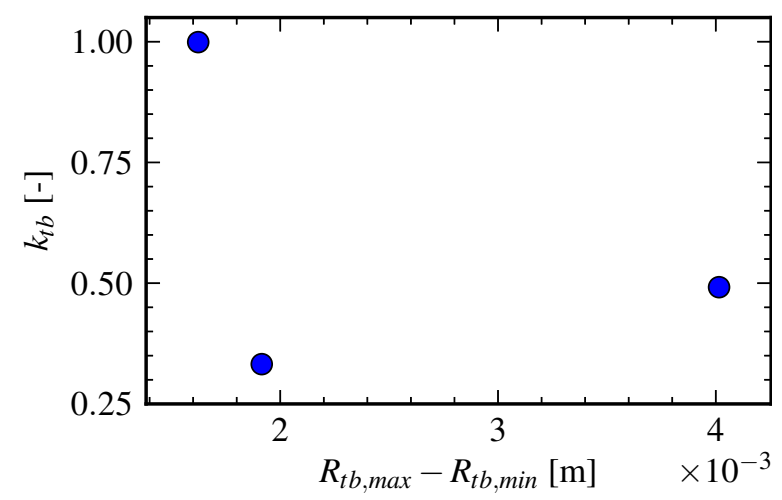

Figure 12: $k_{t b}$ vs $R_{t b, \text { max }}-R_{t b, \text { min }}$

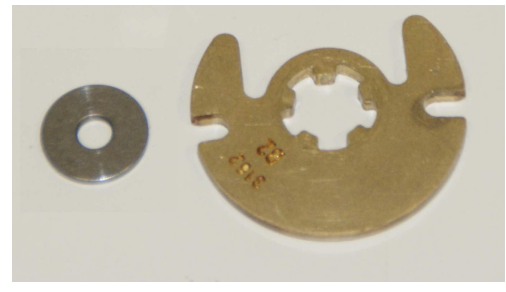

Figure 13: Thrust bearing and washer, first and second turbocharger type

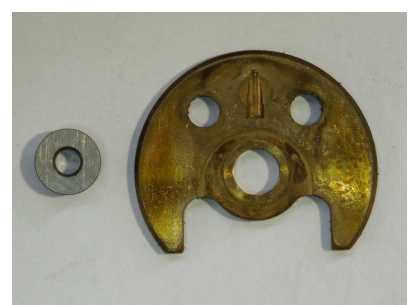

Figure 14: Thrust bearing and washer, third turbocharger type 


\begin{tabular}{|c|c|c|c|}
\hline \multicolumn{4}{|c|}{ Radial bearing only } \\
\hline & First & Second & Third \\
\hline$k_{j b}$ & 0.447 & 0.793 & 0.359 \\
\hline$k_{t b}$ & 0 & 0 & 0 \\
\hline$k_{A_{c}}$ & 0 & 0 & 0 \\
\hline$k_{A_{t}}$ & 0 & 0 & 0 \\
\hline \multicolumn{4}{|c|}{ Thrust bearing only } \\
\hline & First & Second & Third \\
\hline$k_{j b}$ & 0 & 0 & 0 \\
\hline$k_{t b}$ & 1.774 & 1.732 & 3.194 \\
\hline$k_{A_{c}}$ & 0.798 & 0.753 & 1.054 \\
\hline$k_{A_{t}}$ & 1.306 & 1.111 & 1.249 \\
\hline \multicolumn{4}{|c|}{ Full model } \\
\hline & First & Second & Third \\
\hline$k_{j b}$ & 0.332 & 0.739 & 0.295 \\
\hline$k_{t b}$ & 0.492 & 0.333 & 1.000 \\
\hline$k_{A_{c}}$ & 0.791 & 0.892 & 1.000 \\
\hline$k_{A_{t}}$ & 1.084 & 1.290 & 0.985 \\
\hline
\end{tabular}

Table 5: Results of the fitting process

\section{Conclusions}

This work presents a mechanical losses model to be used on turbochargers. The model is based on the Navier Stokes equations applied to the two kinds of bearings (journal and thrust) normally used on this type of element. Making some assumptions a simplified model for the two bearings has been obtained. The models use geometrical characteristics of the bearings and four experimental parameters (one for the radial bearing and three for the axial bearing). These parameters have been adjusted by using quasi-adiabatic tests performed on two different turbochargers, showing that:

- Mechanical efficiency in quasi-adiabatic conditions has a rather linear relationship with turbocharger speed, so at high speeds, it is possible to consider a constant value (close to unity) of this efficiency without affecting the results.

- The journal bearing model gives good results without the need of an axial bearing model at low pressure ratios, but fails as the axial load rises at higher turbocharger speeds.

- The axial bearing model alone is not able to reproduce at all the experimental behavior.

- In order to get the best results, the mechanical losses have to be computed for both the journal and thrust bearings.

- Simple trends have been obtained between fitting coefficients of the model and basic geometrical parameters of the turbocharger. Nevertheless, these simple trends must be confirmed with further experiments in a higher number of different turbocharger models. 


\section{Appendix A. Model description}

Appendix A.1. Journal bearing model

To obtain $\frac{\partial u_{\theta}}{\partial r}$, the continuity equation and the conservation of momentum are used. First, solving the continuity equation for the simplified geometry of figure 4

$$
\frac{\partial \rho}{\partial t}+\frac{1}{r}\left[\frac{\partial\left(\rho r u_{r}\right)}{\partial r}+\frac{\partial\left(\rho u_{\theta}\right)}{\partial \theta}+\frac{\partial\left(\rho r u_{x}\right)}{\partial x}\right]=0
$$

Since the fluid is incompressible (assumption 1) and there is circumferential symmetry (assumption 4):

$$
O\left(\frac{u_{r_{c}}}{h_{j b}}\right)+O\left(\frac{u_{x_{c}}}{L_{j b}}\right)=0 \Rightarrow \frac{u_{r_{c}}}{u_{x_{c}}} \simeq \frac{h_{j b}}{L_{j b}} \ll 1
$$

where big $\mathrm{O}$ notation is used.

This data may be used to get $\frac{\partial u_{\theta}}{\partial r}$ from the conservation of momentum:

$$
\rho\left(\frac{\partial \vec{u}}{\partial t}+\vec{u} \cdot \nabla \vec{u}\right) \cdot \vec{e}_{\theta}=\left(-\nabla p+\nabla \cdot \tau^{\prime}+\rho \vec{f}_{m}\right) \cdot \vec{e}_{\theta}
$$

Taking into account that the flow is incompressible (assumption 1), circumferentially symmetric (assumption 4) and there are no body forces (assumption 5, equation A.3 can be simplified to:

$$
\begin{array}{r}
u_{r} \frac{\partial u_{\theta}}{\partial r}+u_{x} \frac{\partial u_{\theta}}{\partial x}+\frac{u_{r} u_{\theta}}{r}= \\
v\left[\frac{1}{r} \frac{\partial}{\partial r}\left(r \frac{\partial u_{\theta}}{\partial r}\right)+\frac{\partial^{2} u_{\theta}}{\partial x^{2}}-\frac{u_{\theta}}{r^{2}}\right]
\end{array}
$$

Taking the order of magnitude of the different terms of equation A.4 and skipping big $\mathrm{O}$ notation for ease of reading:

$$
\begin{gathered}
u_{r_{c}} \frac{u_{\theta_{c}}}{h_{j b}}+u_{x_{c}} \frac{u_{\theta_{c}}}{L_{j b}}+u_{r_{c}} \frac{u_{\theta_{c}}}{R_{j b}}= \\
v \frac{u_{\theta_{c}}}{h_{j b}^{2}}+v \frac{u_{\theta_{c}}}{L_{j b}^{2}}+v \frac{u_{\theta_{c}}}{R_{j b}^{2}}
\end{gathered}
$$

Dividing by the first term at the right hand side of the equation and using equation A.2.

$$
\begin{gathered}
\frac{\rho u_{x_{c}}}{\mu L_{j b}} h_{j b}^{2}+\frac{\rho u_{x_{c}}}{\mu L_{j b}} h_{j b}^{2}+\frac{\rho u_{x_{c}}}{\mu L_{j b}} h_{j b}^{2} \frac{h_{j b}}{R_{j b}}= \\
1+\left(\frac{h_{j b}}{L_{j b}}\right)^{2}+\left(\frac{h_{j b}}{R_{j b}}\right)^{2}
\end{gathered}
$$

Thus, combining equations A.4 and A.6 and taking into account assumptions 5 and 6, the most important part of the conservation of momentum for the $\theta$ component is:

$$
\frac{1 \partial}{r}\left(r \frac{\partial u_{\theta}}{\partial r}\right)=\left.0 \Rightarrow \frac{\partial u_{\theta}}{\partial r}\right|_{R_{j b}} \simeq n \frac{R_{j b}}{h_{j b}}
$$




\section{Appendix A.2. Thrust bearing model}

The continuity equation gives similar results as in the ideal journal bearing case, thus allowing to simplify even more the momentum equation that will be used to compute the velocity gradient at the thrust bearing. Taking orders of magnitude in equation A.1 in the thrust bearing:

$$
O\left(\frac{u_{r_{c}}}{R_{t b}}\right)+O\left(\frac{u_{x_{c}}}{h_{t b}}\right)=0 \Rightarrow \frac{u_{x_{c}}}{u_{r_{c}}} \simeq \frac{h_{t b}}{R_{t b}} \ll 1
$$

With these results, and taking orders of magnitude in equation A.4 applied to the thrust bearing:

$$
\begin{gathered}
u_{r_{c}} \frac{u_{\theta_{c}}}{R_{t b}}+u_{x_{c}} \frac{u_{\theta_{c}}}{h_{t b}}+u_{r_{c}} \frac{u_{\theta_{c}}}{R_{t b}}= \\
v u_{\theta_{c}}\left(\frac{1}{R_{t b}^{2}}+\frac{1}{h_{t b}^{2}}+\frac{1}{R_{t b}^{2}}\right)
\end{gathered}
$$

Multiplying equation A.9 by $h_{t b}^{2} /\left(v u_{\theta_{c}}\right)$ and using equation A.8.

$$
\begin{gathered}
\frac{\rho u_{r_{c}}}{\mu R_{t b}} h_{t b}^{2}+\frac{\rho u_{x_{c}}}{\mu R_{t b}} h_{t b}^{2}+\frac{\rho u_{r_{c}}}{\mu R_{t b}} h_{t b}^{2}= \\
\left(\frac{h_{t b}}{R_{t b}}\right)^{2}+1+\left(\frac{h_{t b}}{R_{t b}}\right)^{2}
\end{gathered}
$$

Every term in the momentum equation for $\theta$ is negligible but $v \frac{\partial^{2} u_{\theta}}{\partial x^{2}}$. Thus, $u_{\theta}$ and its gradient can be obtained as:

$$
\frac{\partial^{2} u_{\theta}}{\partial x^{2}}=\left.0 \Rightarrow \frac{\partial u_{\theta}}{\partial x}\right|_{\bar{R}_{t b}, h_{t b}}=n \cdot \frac{\bar{R}_{t b}}{h_{t b}}
$$

In this case, the oil film thickness should not be considered constant. To compute it, the radial equation of momentum for steady, incompressible, circumferentially symmetric flow with no body forces is used:

$$
\begin{gathered}
u_{r} \frac{\partial u_{r}}{\partial r}+u_{x} \frac{\partial u_{r}}{\partial x}-\frac{u_{\theta}^{2}}{r}= \\
-\frac{\partial p}{\partial r}+\frac{v}{r} \frac{\partial}{\partial r}\left(r \frac{\partial u_{r}}{\partial r}\right)+v \frac{\partial^{2} u_{r}}{\partial x^{2}}-v \frac{u_{r}}{r^{2}}
\end{gathered}
$$

The orders of magnitude of the different terms are:

$$
\begin{gathered}
u_{r_{c}} \frac{u_{r_{c}}}{R_{t b}}+u_{x_{c}} \frac{u_{r_{c}}}{h_{t b}}+\frac{u_{\theta_{c}}^{2}}{R_{t b}}= \\
\frac{(\Delta p)_{R_{t b}}}{R_{t b}}+v \frac{u_{r_{c}}}{R_{t b}^{2}}+v \frac{u_{r_{c}}}{h_{t b}^{2}}+v \frac{u_{r_{c}}}{R_{t b}^{2}}
\end{gathered}
$$

where $(\Delta p)_{R_{t b}}$ is the variation of the oil pressure through the radius of the thrust bearing. Dividing by the third term on the right hand side of equation A.13.

$$
\begin{aligned}
& \frac{\rho u_{r_{c}}}{\mu R_{t b}} h_{t b}^{2}+\frac{\rho u_{x_{c}}}{\mu R_{t b}} h_{t b}^{2}+\frac{\rho u_{\theta_{c}}}{\mu R_{t b}} h_{t b}^{2} \frac{u_{\theta_{c}}}{u_{r_{c}}}= \\
& \frac{\rho(\Delta p)_{R_{t b}}}{\mu R_{t b} u_{r c}} h_{t b}^{2}+\left(\frac{h_{t b}}{R_{t b}}\right)^{2}+1+\left(\frac{h_{t b}}{R_{t b}}\right)^{2}
\end{aligned}
$$

All the terms are negligible but the first and the third at the right hand side of equation A.14 and hence equation A.12 is simplified to: 


$$
\frac{\partial p}{\partial r}=v \frac{\partial^{2} u_{r}}{\partial x^{2}}
$$

Supposing that the oil pressure is a constant at both sides of the thrust bearing plus a gradient at the working side and integrating twice:

$$
\begin{aligned}
F_{a t} & =\int_{R_{t b, \text { min }}}^{R_{t b, \text { max }}} \int_{0}^{R_{t b, i}} \frac{\partial p}{\partial r} \mathrm{~d} r \mathrm{~d} R_{t b, i} \\
& =\int_{R_{t b, \text { min }}}^{R_{t b, \text { max }}} \int_{0}^{R_{t b, i}} v \frac{\partial^{2} u_{r}}{\partial x^{2}}
\end{aligned}
$$

$u_{r}$ can be computed supposing a parabolic radial speed profile, similar to that of a Poiseuille planar flow:

$$
u_{r}(x)=\frac{3}{2} \bar{u}_{r} \cdot\left(\frac{2 x}{h_{t b}}-1\right)^{2}
$$

The mean radial speed at a given radius is modelled as:

$$
\bar{u}_{r}=\frac{\dot{m}_{t b}}{2 \pi r \rho h_{t b}}
$$

The mass flow rate through the ideal thrust bearing can be computed as a constant fraction of the total mass flow rate in the full bearing system:

$$
\dot{m}_{t b}=k_{m} \dot{m}
$$

Using equations A.17 A.18 and A.19together:

$$
u_{r}(x)=\frac{3 k_{m} \dot{m}}{4 \pi r \rho h_{t b}} \cdot\left(\frac{2 x}{h_{t b}}-1\right)^{2}
$$

Using equation A.20 in equation A.16 and solving for $h_{t b}$ :

$$
h_{t b} \simeq \sqrt[3]{\frac{6 k_{m} \dot{m} \varphi \mu}{F_{a t} \rho}}
$$

where:

$$
\varphi=\frac{R_{t b, \text { max }}^{2}}{2} \cdot \log \left(\frac{R_{t b, \text { max }}}{R_{t b, \text { min }}}\right)+\frac{\left(R_{t b, \text { min }}^{2}-R_{t b, \text { max }}^{2}\right)}{4}
$$

The axial thrust can be computed using geometrical information and pressures at the compressor and turbine wheels. As the thrust bearing can be modelled symmetrically, only the absolute magnitude of the force is needed. Figure A.1 shows an scheme of the proposed pressure distribution linealization at turbine and compressor sides.

The pressure at the outlet of the compressor wheel $\left(p_{2}^{\prime}\right)$ is supposed as the mean between the pressure at the inlet $\left(p_{1}\right)$ and the pressure at the outlet $\left(p_{2}\right)$ of the compressor. The pressure at the inlet of the turbine wheel can be calculated as seen in [22].

$$
\begin{aligned}
F_{\text {comp }} & =A_{\text {comp }}^{\prime} \cdot\left(\frac{p_{1}+\frac{p_{1}+p_{2}}{2}}{2}-\frac{p_{1}+p_{2}}{2}\right) \\
& =A_{\text {comp }}^{\prime} \cdot \frac{p_{1}-p_{2}}{4}
\end{aligned}
$$




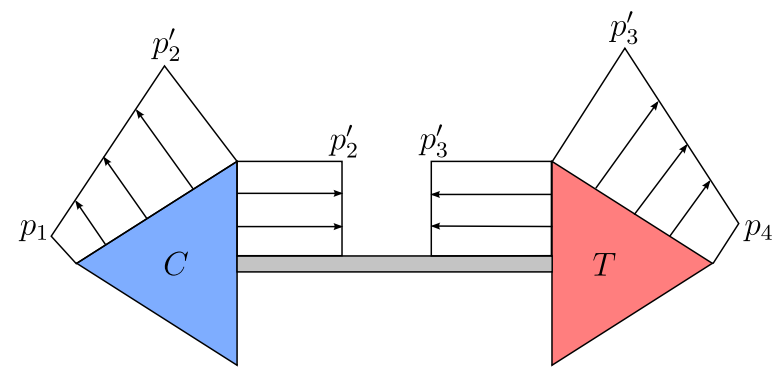

Figure A.1: Schematic pressure distribution at the compressor and turbine wheels

$$
\begin{aligned}
F_{\text {turb }}= & A_{\text {turb }}^{\prime} \cdot\left(p_{3}^{\prime}-\frac{p_{3}^{\prime}+p_{4}}{2}\right)=A_{\text {turb }}^{\prime} \cdot\left(\frac{p_{3}^{\prime}-p_{4}}{2}\right) \\
F_{a t} & =\left|F_{\text {comp }}+F_{\text {turb }}\right| \\
& =\left|A_{\text {comp }}^{\prime} \frac{p_{1}-p_{2}}{4}+A_{\text {turb }}^{\prime} \frac{p_{3}^{\prime}-p_{4}}{2}\right|
\end{aligned}
$$

where $A_{\text {comp }}^{\prime}$ and $A_{\text {turb }}^{\prime}$ are the effective areas of the compressor and the turbine wheels. Each one is the projected area in the axial direction, multiplied by a constant that takes into account the non-linearities of the pressure distribution in the wheels:

$$
\begin{gathered}
A_{\text {comp }}^{\prime}=k_{A_{\text {comp }}} A_{\text {comp }} \\
A_{\text {turb }}^{\prime}=k_{A_{\text {turb }}} A_{\text {turb }}
\end{gathered}
$$

The value of the tangential speed gradient in the thrust bearing is computed in equation A.28.

$$
\left.\frac{\partial u_{\theta}}{\partial x}\right|_{\bar{R}_{t b}, h_{t b}}=n \cdot \frac{\bar{R}_{t b}}{\sqrt[3]{\frac{6 k_{m} \dot{m} \varphi \mu}{F_{a t} \rho}}}
$$

where $F_{a t}$ is shown in equation A.25.

\section{Nomenclature}

Symbols.
$A$ area
c specific heat capacity
$\vec{e}$ unit vector
$\overrightarrow{f_{m}}$ body forces
$h$ oil film thickness
$k$ proportionality constant
$L$ length 
$\dot{m}$ mass flow rate

$n$ rotational speed

$\vec{n}$ normal unitary vector

$p$ pressure

$\dot{Q}$ heat flow

$R$ radius

$r$ radial coordinate

$T$ temperature

$t$ time

$u$ speed

$\dot{W}$ power

$x$ axial coordinate

Greek letters.

$\eta$ efficiency

$\mu$ dynamic viscosity

$v$ kinematic viscosity

$\varphi$ geometrical parameter

$\rho$ density

$\tau^{\prime}$ viscous stresses tensor

$\theta$ circumferential coordinate

Subscripts.

at axial thrust

$c$ characteristic

comp compressor

$i$ component

in inlet

$j b$ journal bearing

$m$ mass flow rate

$\max$ maximum quantity

min minimum quantity

$m l$ mechanical losses 
oil oil

$T$ temperature

turb turbine

$t b$ thrust bearing

1 compressor inlet

2 compressor outlet

3 turbine inlet

4 turbine outlet

\section{Acknowledgements}

The authors of this paper wish to thank M.A. Ortiz and V. Ucedo for their invaluable work during the experimental setup and campaign, F.J. Arnau, Ph.D, for its excellent job maintaining and expanding OpenWAMs code base and M.A. Reyes-Belmonte for all his hard and rigorous work extrapolating turbine maps and preparing and launching the simulations.

\section{References}

[1] EEC, Regulation (ec) no 595/2009 of the european parliament and of the council, Official Journal of the European Communities, 2009.

[2] N. Baines, Fundamentals of Turbocharging, Concepts NREC, 2005.

[3] A. Diango, C. Perilhon, G. Descombes, E. Danho, Application of exergy balances for the optimization of non-adiabatic small turbomachines operation, Energy 36 (2011) 2924-2936.

[4] M. Deligant, P. Podevin, G. Descombes, Cfd model for turbocharger journal bearing performances, Applied Thermal Engineering 31 (2011) 811-819.

[5] L. Lihua, S. Hao, L. Y. Z. Qiang, Research on static stiffness of hydrostatic bearing using fluid-structure interaction analysis, Procedia Engineering 29 (2012) 1304-1308.

[6] W. J. Chen, Rotordynamics and bearing design of turbochargers, Mechanical Systems and Signal Processing 29 (2012) 77-89.

[7] S. Shaaban, Experimental Investigation and Extended Simulation of Turbocharger Non-Adiabatic Performance, Ph.D. thesis, Universitt Hannover, Fachbereich Maschinenbau, 2004.

[8] P. Podevin, A. Clenci, G. Descombes, Influence of the lubricating oil pressure and temperature on the performance at low speeds of a centrifugal compressor for an automotive engine, Applied Thermal Engineering 31 (2011) 194 - 201.

[9] F. Payri, J. R. Serrano, P. Olmeda, A. Páez, F. Vidal, Experimental methodology to characterize mechanical losses in small turbochargers, ASME Conference Proceedings 2010 (2010) 413-423.

[10] L. Hu, C. Yang, H. Sun, E. Krivizky, L. Larosiliere, J. Zhang, M. Lai, Experimental and computational analysis of impact of self recirculation casing treatment on turbocharger compressor, SAE Technical papers (2010) 2010-01-1224.

[11] M. Deligant, P. Podevin, G. Descombes, Experimental identification of turbocharger mechanical friction losses, Energy 39 (2012) $388-394$.

[12] S. of Automotive Engineers, Supercharger testing standard, SAE SAE J1723 (1995).

[13] S. of Automotive Engineers Inc, Turbocharger gas stand test code, SAE SAE J1826 (1995).

[14] N. Baines, K. D. Wygant, A. Dris, The Analysis of Heat Transfer in Automotive Turbochargers, Journal of Engineering for Gas Turbineas and Power - Transactions of the ASME 132 (2010). ASME Gas Turbine Technical Congress and Exposition, Orlando, FL, JUN 08-12, 2009.

[15] M. Cormerais, J. Hetet, P. Chessé, A. Malboom, Heat transfers characterization in a variable geometry turbocharger: Experiments and correlations, Proceeding of ASME Internal Combustion Engine Division (2006).

[16] J. R. Serrano, P. Olmeda, A. Páez, F. Vidal, An experimental procedure to determine heat transfer properties of turbochargers, Measurement Science and Technology 21 (2010) 035109.

[17] J. R. Serrano, F. J. Arnau, P. Fajardo, M. A. Reyes-Belmonte, Contribution to the modeling and understanding of cold pulsating flow influence in the efficiency of small radial turbines for turbochargers, Journal of Engineering for Gas Turbines and Power (2012).

[18] M. V. Casey, M. Schlegel, Estimation of the performance of turbocharger compressors at extremely low pressure ratios, Proceedings of the Institution of Mechanical Engineers Part A - Journal of Power and Energy 224 (2010) 239-250.

[19] A. J. Torregrosa, P. Olmeda, J. Martín, C. Romero, A Tool for Predicting the Thermal Performance of a Diesel Engine, Heat Transfer Engineering 32 (2011) 891-904.

[20] D. Kraft, DFVLR-FB., A Software Package for Sequential Quadratic Programming, Forschungsbericht // Deutsche Forschungs- und Versuchsanstalt für Luft- und Raumfahrt, DFVLR, 1988.

[21] E. Jones, T. Oliphant, P. Peterson, et al., SciPy: Open source scientific tools for Python, http://www.scipy.org/ 2001-.

[22] J. R. Serrano, F. J. Arnau, V. Dolz, A. Tiseira, C. Cervelló, A model of turbocharger radial turbines appropriate to be used in zero- and onedimensional gas dynamics codes for internal combustion engines modelling, Energy Conversion and Management 49 (2008) 3729-3745. 


\section{Figures caption}

Figure 1. Schematic test bench and location of main sensors.

Figure 2. Quasi-adiabatic measurement range in compressor map.

Figure 3 . Measured mechanical efficiencies.

Figure 4 . Schematic model of a journal bearing.

Figure 5. Schematic model of a thrust bearing.

Figure 6 . Simplified model of a short journal bearing.

Figure 7. Simplified model of a short thrust bearing.

Figure 8 Model results applied to full dataset. The left column is adjusted with no radial bearing effects. The right column is adjusted with no axial bearing effects.

Figure 9 Model results applied to full dataset. The parameters are obtained fitting only a random subset of the experimental data.

Figure 10 Model results applied to full dataset. The parameters are obtained fitting the whole dataset.

Figure 11 Correlation between the journal bearing model and $A_{c}$.

Figure $12 k_{t b}$ vs $R_{t b, \text { max }}-R_{t b, \text { min }}$.

Figure 13 Thrust bearing and washer, first and second turbocharger type.

Figure 14 Thrust bearing and washer, third turbocharger type.

Figure A.1. Schematic pressure distribution at the compressor and turbine wheels. 